\title{
On an Invariant Set in the Heat Conductivity Problem with Time Lag
}

\author{
M. Tukhtasinov, ${ }^{1}$ G. I. Ibragimov, ${ }^{2}$ and N. O. Mamadaliev ${ }^{1}$ \\ ${ }^{1}$ National University of Uzbekistan, Tashkent 100174, Uzbekistan \\ ${ }^{2}$ Universiti Putra Malaysia, 43400 Serdang, Malaysia \\ Correspondence should be addressed to G. I. Ibragimov; ibragimov@upm.edu.my
}

Received 5 September 2013; Accepted 20 November 2013

Academic Editor: Pavel Kurasov

Copyright (C) 2013 M. Tukhtasinov et al. This is an open access article distributed under the Creative Commons Attribution License, which permits unrestricted use, distribution, and reproduction in any medium, provided the original work is properly cited.

The problems of weak and strong invariance of a constant multivalued mapping with respect to the heat conductivity equation with time lag are studied. Sufficient conditions of weak and strong invariance of a given multivalued mapping are obtained.

\section{Introduction}

1.1. Related Works. There are many theoretical and practical problems in control problems with distributed parameters where known methods do not work to solve them. The typical examples of such problems are conservation of temperature of a volume within admissible bounds and deviation from undesirable states.

Note that the works [1-7] were devoted to the problems of invariance of given sets for the controlled systems. In these works, some results on construction of core of liveness, the maximal weak invariant subset of the given set was obtained for the control system.

In the paper [3], a family of trajectories, which is kept to be within the given set until a certain time (viability), is analytically described for control systems given by differential inclusions. The paper [8] deals with the problem of diffusion process control by boundary control.

However, all the works mentioned above relate to control systems with concentrated parameters. In the papers $[9,10]$, weak and strong invariance of the given set with respect to a system with distributed parameters were studied. Alimov [11], Albeverio and Alimov [12] studied interesting applied control problems on heat distribution by convectors in a volume.

The present paper deals with the problems of weak and strong invariance of given multivalued mapping for the 3rd heat conductivity boundary value problem with time lag. In the equation of this problem, the control parameter appears on the right hand side. We obtain conditions which can be easily checked to determine the invariance of the given constant multivalued mapping.

1.2. Preliminaries. First of all, we recall some definitions. A bounded region $\Omega\left(\subset \mathbb{R}^{n}\right)$ is referred to as the region with piecewise smooth boundary if its boundary $\Gamma=\bar{\Omega} \backslash \Omega$ can be represented as follows: $\Gamma=\sum_{j=1}^{N} \bar{\Gamma}_{j}$, where $\Gamma_{j} \subset \Gamma$ is an open set with respect to the topology on $\Gamma$ induced by the topology in $\mathbb{R}^{n}$. Moreover, each $\Gamma_{j}$ is a connected surface of class $C^{1}$; that is, for any point $x_{0} \in \Gamma_{j}$ there exists a ball $U_{\epsilon}\left(x_{0}\right)$ of radius $\epsilon>0$ such that the piece $\Gamma_{j} \cap U_{\epsilon}\left(x_{0}\right)$ of the surface is given by the equation of the form $x_{k}=f_{k}\left(x_{1}, \ldots, x_{k-1}, x_{k+1}, \ldots, x_{n}\right)$, where $f_{k}(\cdot) \in C^{1}$ and $1 \leq k \leq n$.

Let $\Omega$ be a region in $\mathbb{R}^{n}$ with piecewise smooth boundary. We will use the letter $A$ to denote the following differential operator $[13,14]$ :

$$
A(x, D) \varphi=-\sum_{i, j=1}^{n} \frac{\partial}{\partial x_{i}}\left(a_{i j}(x) \frac{\partial \varphi}{\partial x_{j}}\right)
$$

where the functions $a_{i j}(x) \in C^{1}(\Omega) \cap C(\bar{\Omega})$ satisfy the conditions $a_{i j}(x)=a_{j i}(x), x \in \Omega$, and

$$
\sum_{i, j=1}^{n} a_{i j}(x) \xi_{i} \xi_{j} \geq \gamma \sum_{i=1}^{n} \xi_{i}^{2}
$$


for all $\left(\xi_{1}, \xi_{2}, \ldots, \xi_{n}\right) \in R^{n}$. The inequality (2) is called the condition of uniform ellipticity of the operator $A$ defined by (1). The domain of the operator $A$ is $C^{2}(\Omega)$, which is the space of functions that are twice continuously differentiable in $\Omega$ and continuous on $\Omega \cup \partial \Omega$.

Define the operator $P$ by the equation

$$
P \varphi=\sum_{i, j=1}^{n} a_{i j}(x) \frac{\partial \varphi}{\partial x_{j}} \cos \left(l, x_{i}\right)+k(x) \varphi, \quad x \in \partial \Omega,
$$

where $l$ denotes the upward unit normal vector on $\partial \Omega$ and $k(x)$ is a given positive continuous function defined on $\partial \Omega$.

Definition 1. The number $\lambda$ for which the following boundary value problem:

$$
\begin{gathered}
A(x, D) \varphi=\lambda \varphi, \quad x \in \Omega, \\
P \varphi=0, \quad x \in \partial \Omega,
\end{gathered}
$$

has nonzero solution $\varphi_{\lambda}(x), x \in \Omega$ is called eigenvalue of this boundary value problem, and the solution $\varphi_{\lambda}(x)$ is called eigenfunction of the boundary value problem.

Since problem (4) is homogeneous, we assume that

$$
\int_{\Omega} \varphi_{\lambda}^{2} d x=1
$$

As the operator (1) is self-adjoint, then it has a discrete spectrum $[13,14]$; that is,

(i) there exist a countable set of eigenvalues $\lambda_{k}$ of the problem (4) such that

$$
0<\lambda_{1} \leq \lambda_{2} \leq \cdots \leq \lambda_{n} \leq \cdots, \quad \lambda_{n} \longrightarrow \infty, n \longrightarrow \infty,
$$

(ii) for each eigenvalue $\lambda_{k}$, there are a finite number of eigenfunctions $\varphi_{k}$ corresponding to it such that $\int_{\Omega} \varphi_{i}(x) \varphi_{j}(x) d x=\delta_{i j}$, where

$$
\delta_{i j}= \begin{cases}1, & \text { if } i=j, \\ 0, & \text { if } i \neq j,\end{cases}
$$

(iii) the set of all eigenvalues $\left\{\varphi_{k}\right\}$ is complete (closed) in the space $L_{2}(\Omega)$; that is, any function $f$ from the space $L_{2}(\Omega)$ can be uniquely represented in the form

$$
f(x)=\sum_{k=1}^{\infty} f_{k} \varphi_{k}(x) \text {, }
$$

where the equality is understood in the following sense:

$$
\int_{\Omega}\left[f(x)-\sum_{k=1}^{N} f_{k} \varphi_{k}(x)\right]^{2} d x \longrightarrow 0, \quad N \longrightarrow \infty .
$$

The Fourier coefficients $f_{k}$ in the Fourier series (8) of $f(x)$ are defined by formula

$$
f_{k}=\int_{\Omega} f(x) \varphi_{k}(x) d x .
$$

Since the operator $A$ is self-adjoint, in certain conditions on the functions $a_{i j}, \varphi$, and $\psi$, the Green formula is written in the form

$$
\begin{gathered}
\int_{\Omega}(\psi A(x, D) \varphi-\varphi A(x, D) \psi) d x \\
=\int_{\partial \Omega}(\psi P \varphi-\varphi P \psi) d S .
\end{gathered}
$$

Using these, we construct the following spaces depending on a parameter. Let $r$ be any nonnegative number. Denote

$$
\begin{gathered}
\ell_{r}=\left\{\alpha=\left(\alpha_{1}, \alpha_{2}, \ldots\right) \mid \sum_{i=1}^{\infty} \lambda_{i}^{r} \alpha_{i}^{2}<\infty\right\}, \\
H_{r}(\Omega)=\left\{f \in L_{2}(\Omega) \mid f=\sum_{i=1}^{\infty} \alpha_{i} \varphi_{i}, \alpha \in \ell_{r}\right\} .
\end{gathered}
$$

We now define inner product and norm in the spaces $\ell_{r}, H_{r}=H_{r}(\Omega)$. Let

$$
\alpha, \beta \in \ell_{r}, \quad f=\sum_{i=1}^{\infty} \alpha_{i} \varphi_{i}, \quad g=\sum_{i=1}^{\infty} \beta_{i} \varphi_{i} .
$$

Set

$$
\begin{array}{cc}
(\alpha, \beta)_{r}=\sum_{i=1}^{\infty} \lambda_{i}^{r} \alpha_{i} \beta_{i}, & \|\alpha\|=(\alpha, \alpha)_{r}^{1 / 2}, \\
(f, g)_{r}=(\alpha, \beta)_{r}, & \|f\|=\|\alpha\| .
\end{array}
$$

It should be noted that $H_{0}(\Omega)=L_{2}(\Omega)$ and $H_{r}(\Omega) \subset H_{s}(\Omega)$ for all $0 \leq s \leq r$.

Denote by $C\left(0, T ; H_{r}(\Omega)\right)$ and $L_{2}\left(0, T ; H_{r}(\Omega)\right)$ the spaces of continuous functions and summable quadratically measurable functions defined on $[0, T]$ with the values in $H_{r}(\Omega)$, respectively, where $T$ is a positive number.

1.3. Auxiliary Statements. Consider the following heat exchange control problem with lag:

$$
\begin{aligned}
& \frac{\partial z(x, t)}{\partial t}+A(x, D) z(x, t) \\
& \quad=z_{0}(x, t-h)+u(x, t), \quad 0<t \leq T,
\end{aligned}
$$

with boundary

$$
P z(x, t)=0, \quad 0 \leq t \leq T, x \in \partial \Omega,
$$

and initial conditions

$$
z(x, 0)=z_{0}(x, 0),
$$


where $z_{0}(x, \cdot) \in X ; X=\left\{z(x, \cdot) \mid z(x, t) \in H_{r},-h \leq\right.$ $t \leq 0\}$. Here, $z(\cdot)=z(x, \cdot)$ and $u(\cdot)=u(x, \cdot)$ are abstract functions whose values at each $t>0$ are unique elements of the space $H_{r}(\Omega) ; h$ is a positive fixed number and $T$ is a positive number.

Further, we use the same letter $A$ to denote the bounded operator that maps $H_{r}$ into $H_{r+2}$ and is defined by the following formula

$$
A \varphi=A\left(\sum_{k=1}^{\infty} c_{k} \varphi_{k}\right)=\sum_{k=1}^{\infty} \lambda_{k} c_{k} \varphi_{k}
$$

where $\varphi=\sum_{k=1}^{\infty} c_{k} \varphi_{k}$. Clearly, we obtain from this that $\|A f\|_{H_{r}}=\|f\|_{H_{r+2}}$.

The problem (15)-(17) is understood in the sense of the theory of generalized functions (the theory of distributions) with the values in $H_{r}$. We will look for the solution of the problem (15)-(17), which is continuous with respect to $t$ and its values belong to one of the spaces $H_{r}$, and the initial and boundary conditions are considered as equality of elements of these spaces.

In the control problem (15)-(17), the control function $u(\cdot)$ is subjected to either constraint

$$
\|u(t)\|_{H_{r}} \leq \rho, \quad 0 \leq t \leq T
$$

or

$$
u(\cdot) \in L_{2}\left([0, h] ; H_{r}\right), \quad\|u(\cdot)\|_{L_{2}\left([0, h] ; H_{r}\right)} \leq \rho .
$$

Accordingly, in this paper, we consider both of the problems (15)-(19) and (15)-(17), (20). The control function $u(\cdot)$ that satisfies either (19) or (20) is called admissible.

Assume that the problem (15)-(17) has a solution $z(t), 0 \leq$ $t \leq h$, at some admissible control $u(\cdot)$. Then we have

$$
\begin{gathered}
z(t)=\sum_{k=1}^{\infty} z_{k}(t) \varphi_{k}(x) ; \\
z_{k}(t)=\int_{\Omega} z(t) \varphi_{k}(x) d x .
\end{gathered}
$$

It follows from (15) that

$$
\begin{array}{r}
\int_{\Omega}\left(\frac{\partial z(t)}{\partial t}+A z(x)-z_{0}(t-h)-u(t)\right) \varphi_{k}(x) d x \equiv 0 \\
k=1,2, \ldots
\end{array}
$$

Also, by definition of the functions $z(t), \varphi_{k}(x)$ we obtain

$$
\begin{gathered}
P z(t)=0, \quad 0 \leq t \leq h, x \in \partial \Omega, \\
P \varphi_{k}=0, \quad A \varphi_{k}=\lambda_{k} \varphi_{k} .
\end{gathered}
$$

Therefore, in view of (11) we have

$$
\int_{\Omega} \varphi_{k}(x) A z(t) d x=\lambda_{k} z_{k}(t) .
$$

\section{Denoting}

$$
\begin{gathered}
z_{0 k}(t-h)=\int_{\Omega} z_{0}(t-h) \varphi_{k}(x) d x, \\
u_{k}(t)=\int_{\Omega} u(t) \varphi_{k}(x) d x
\end{gathered}
$$

and using the fact that

$$
\int_{\Omega} \frac{\partial z(t)}{\partial t} \varphi_{k}(x) d x=\frac{d z_{k}(t)}{d t},
$$

we deduce from formulas (22) and (24) that

$$
\begin{array}{r}
\dot{z}_{k}(t)+\lambda_{k} z_{k}(t)=z_{0 k}(t-h)+u_{k}(t), \\
0 \leq t \leq h, \quad k=1,2, \ldots
\end{array}
$$

Since the function (21) must satisfy the initial condition $z(0)=z_{0}(0)$, we have

$$
z_{k}(0)=z_{0 k}^{0}=\int_{\Omega} z_{0}(0) \varphi_{k}(x) d x, \quad k=1,2, \ldots
$$

From this and (27) we see that

$$
\begin{array}{r}
z_{k}(t)=e^{-\lambda_{k} t} z_{0 k}^{0}+\int_{0}^{t} e^{-\lambda_{k}(t-\tau)}\left(z_{0 k}(\tau-h)+u_{k}(\tau)\right) d \tau, \\
k=1,2, \ldots
\end{array}
$$

Thus, (21) and (29) define the formal solution of the problem $(15)-(17)$ on $[0, h]$ which can be written as follows:

$$
\begin{aligned}
& z(t) \\
& =\sum_{k=1}^{\infty}\left[e^{-\lambda_{k} t} z_{0 k}^{0}+\int_{0}^{t} e^{-\lambda_{k}(t-\tau)}\left(z_{0 k}(\tau-h)+u_{k}(\tau)\right) d \tau\right] \\
& \quad \times \varphi_{k}(x) .
\end{aligned}
$$

The following assumption will be needed throughout the paper.

Assumption 2. Let functions $z(\cdot)$ of the set $X$ satisfy the following conditions:

$$
\begin{gathered}
\sum_{k=1}^{\infty} \lambda_{k}^{r} \int_{0}^{t}\left|z_{0 k}(\tau-h)\right|^{2} d \tau \leq b^{2}, \\
\sum_{k=1}^{\infty} \lambda_{k}^{r}\left|z_{0 k}(0)\right|^{2} \leq b^{2}, \\
\sum_{k=1}^{\infty} \lambda_{k}^{r} \int_{0}^{t} e^{-\lambda_{k}(t-\tau)}\left|z_{0 k}(\tau-h)\right|^{2} d \tau \\
\leq b^{2} \int_{0}^{t} e^{-\lambda_{1}(t-\tau)} d \tau, \quad 0 \leq t \leq h .
\end{gathered}
$$


Proposition 3. For any function $f(t),\|f(t)\|_{H_{r}} \leq M, 0 \leq t \leq$ $T$, the following inequality holds:

$$
\begin{gathered}
\sum_{k=1}^{\infty} \lambda_{k}^{r}\left(\int_{0}^{t} e^{-\lambda_{k}(t-\tau)} f_{k}(\tau) d \tau\right)^{2} \leq a_{1}^{2}(t) M^{2} ; \\
a_{1}(t)=\frac{1-e^{-\lambda_{1} t}}{\lambda_{1}}, \quad 0 \leq t \leq T,
\end{gathered}
$$

where $\lambda_{1}, \lambda_{2}, \ldots$ are the eigenvalues of the operator $A$ arranged in decreasing order, $M$ is a positive number, and $f_{k}(\cdot)$ is the Fourier coefficient of the function $f(t)$.

Proof. Indeed, for $0 \leq t \leq T$, using the Cauchy-Schwartz inequality and the fact that

$$
\|f(\tau)\|_{H_{r}}^{2}=\sum_{k=1}^{\infty} \lambda_{k}^{r} f_{k}^{2}(\tau) \leq M^{2}, \quad 0 \leq \tau \leq t \leq T,
$$

we obtain

$$
\begin{aligned}
\sum_{k=1}^{\infty} \lambda_{k}^{r}\left(\int_{0}^{t} e^{-\lambda_{k}(t-\tau)} f_{k}(\tau) d \tau\right)^{2} \\
\quad=\sum_{k=1}^{\infty} \lambda_{k}^{r}\left(\int_{0}^{t} e^{-\left(\lambda_{k} / 2\right)(t-\tau)} \cdot e^{-\left(\lambda_{k} / 2\right)(t-\tau)} f_{k}(\tau) d \tau\right)^{2} \\
\quad \leq \sum_{k=1}^{\infty} \lambda_{k}^{r} \int_{0}^{t} e^{-\lambda_{1}(t-\tau)} d \tau \int_{0}^{t} e^{-\lambda_{1}(t-\tau)} f_{k}^{2}(\tau) d \tau \\
\quad \leq \int_{0}^{t} e^{-\lambda_{1}(t-\tau)} d \tau \int_{0}^{t} e^{-\lambda_{1}(t-\tau)} \cdot \sum_{k=1}^{\infty} \lambda_{k}^{r} f_{k}^{2}(\tau) d \tau \\
\quad \leq a_{1}(t) \int_{0}^{t} e^{-\lambda_{1}(t-\tau)} M^{2} d \tau=a_{1}^{2}(t) M^{2} .
\end{aligned}
$$

Theorem 4. Let $u(t), 0 \leq t \leq h$ be an admissible control that satisfies the condition (19), and let Assumption 2 be satisfied. Then there exists a unique solution of the problem (15)-(17) in the space $C\left([0, h] ; H_{r}(\Omega)\right)$.

Proof. To prove the theorem we use the formal representation (30) of the solution of the problem (15)-(17). By definition of the norm in $H_{r}(\Omega)$ for $0 \leq t \leq h$, we have

$$
\begin{aligned}
\|z(t)\|_{H_{r}}^{2} & =\sum_{k=1}^{\infty} \lambda_{k}^{r}\left|z_{k}(t)\right|^{2} \\
& =\sum_{k=1}^{\infty} \lambda_{k}^{r}\left(e^{-\lambda_{k} t} z_{0 k}^{0}+\int_{0}^{t} e^{-\lambda_{k}(t-\tau)} f_{k}(\tau) d \tau\right)^{2},
\end{aligned}
$$

where $f_{k}(\tau)=z_{0 k}(\tau-h)+u_{k}(\tau)$.
We use the Cauchy-Schwartz inequality to obtain the following chain of relations:

$$
\begin{aligned}
\sum_{k=1}^{\infty} \lambda_{k}^{r} & \left(\int_{0}^{t} e^{-\lambda_{k}(t-\tau)} f_{k}(\tau) d \tau\right)^{2} \\
& \leq \sum_{k=1}^{\infty} \lambda_{k}^{r}\left(\int_{0}^{t} e^{-\left(\lambda_{k}(t-\tau)\right) / 2} e^{-\left(\lambda_{k}(t-\tau)\right) / 2} f_{k}(\tau) d \tau\right)^{2} \\
& \leq \sum_{k=1}^{\infty} \lambda_{k}^{r} \int_{0}^{t} e^{-\lambda_{k}(t-\tau)} d \tau \int_{0}^{t} e^{-\lambda_{k}(t-\tau)} f_{k}^{2}(\tau) d \tau \\
& \leq \sum_{k=1}^{\infty} \lambda_{k}^{r} \frac{1-e^{-\lambda_{k} t}}{\lambda_{k}} \int_{0}^{t} e^{-\lambda_{k}(t-\tau)} f_{k}^{2}(\tau) d \tau .
\end{aligned}
$$

Therefore,

$$
\begin{aligned}
\sum_{k=1}^{\infty} \lambda_{k}^{r} & \frac{1-e^{-\lambda_{k} t}}{\lambda_{k}} \int_{0}^{t} e^{-\lambda_{k}(t-\tau)} f_{k}^{2}(\tau) d \tau \\
& \leq a_{1}(t) \sum_{k=1}^{\infty} \lambda_{k}^{r} \int_{0}^{t} e^{-\lambda_{k}(t-\tau)}\left(z_{0 k}(\tau-h)+u_{k}(\tau)\right)^{2} d \tau .
\end{aligned}
$$

Next, we expand the integrand on the right hand side of the last inequality; then we use the Cauchy-Schwartz inequality and after that we use Assumption 2 to obtain

$$
\sum_{k=1}^{\infty} \lambda_{k}^{r}\left(\int_{0}^{t} e^{-\lambda_{k}(t-\tau)} f_{k}(\tau) d \tau\right)^{2} \leq(b+\rho)^{2} a_{1}^{2}(t) .
$$

Expanding the square in (36), and then using (39) and Assumption 2, we arrive at

$$
\|z(t)\|_{H_{r}}^{2} \leq\left(e^{-\lambda_{1} t} b+(b+\rho) a_{1}(t)\right)^{2} .
$$

Hence, $z(t) \in H_{r}(\Omega)$ for each $t \in[0, h]$.

We now show that the function $z(t), 0 \leq t \leq h$, is continuous with respect to the norm of the space $H_{r}$. We have

$$
\begin{aligned}
\|z(t+\delta)-z(t)\|_{H_{r}}^{2}= & \sum_{k=1}^{\infty} \lambda_{k}^{r}\left|z_{k}(t+\delta)-z_{k}(t)\right|^{2} \\
= & \sum_{k=1}^{N} \lambda_{k}^{r}\left|z_{k}(t+\delta)-z_{k}(t)\right|^{2} \\
& +\sum_{k=N+1}^{\infty} \lambda_{k}^{r}\left|z_{k}(t+\delta)-z_{k}(t)\right|^{2},
\end{aligned}
$$

where the numbers $\delta$ and $N$ will be chosen depending on any $\varepsilon>0$.

By (29), We have

$$
\begin{aligned}
z_{k}(t+\delta)-z_{k}(t)= & z_{0 k}^{0} e^{-\lambda_{k} t}\left(e^{-\lambda_{k} \delta}-1\right) \\
& +\int_{0}^{t} e^{-\lambda_{k}(t-\tau)}\left(e^{-\lambda_{k} \delta}-1\right) f_{k}(\tau) d \tau \\
& +\int_{t}^{t+\delta} e^{-\lambda_{k}(t+\delta-\tau)} f_{k}(\tau) d \tau .
\end{aligned}
$$


Combining this with (41), we obtain

$$
\begin{gathered}
\|z(t+\delta)-z(t)\|_{H_{r}}^{2} \leq I_{1}+I_{2}+I_{3} \\
I_{1}=3 \sum_{k=1}^{N} \lambda_{k}^{r}\left|e^{-\lambda_{k} \delta}-1\right|^{2}\left(\left|z_{0 k}^{0}\right|^{2}+\left|\int_{0}^{t} e^{-\lambda_{k}(t-\tau)} f_{k}(\tau) d \tau\right|^{2}\right) \\
I_{2}=3 \sum_{k=N+1}^{\infty} \lambda_{k}^{r}\left(\left|z_{0 k}^{0}\right|^{2}+\left|\int_{0}^{t} e^{-\lambda_{k}(t-\tau)} f_{k}(\tau) d \tau\right|^{2}\right) \\
I_{3}=3 \sum_{k=1}^{\infty} \lambda_{k}^{r}\left|\int_{t}^{t+\delta} e^{-\lambda_{k}(t+\delta-\tau)} f_{k}(\tau) d \tau\right|^{2} .
\end{gathered}
$$

Let $\varepsilon$ be an arbitrary positive number. Since the series in (31) and (39) are convergent, one can choose the number $N$ so that $I_{2}<\varepsilon^{2} / 3$. Then, we choose $\delta$ so that $I_{1}<\varepsilon^{2} / 3$. Further, similar to (39) we have

$$
\begin{aligned}
\sum_{k=1}^{\infty} \lambda_{k}^{r} & \left(\int_{t}^{t+\delta} e^{-\lambda_{k}(t+\delta-\tau)} f_{k}(\tau) d \tau\right)^{2} \\
& \leq e^{-2 \lambda_{1} \delta} \sum_{k=1}^{\infty} \lambda_{k}^{r}\left(\int_{t}^{t+\delta} e^{-\lambda_{k}(t-\tau)} f_{k}(\tau) d \tau\right)^{2} \\
& \leq e^{-2 \lambda_{1} \delta}\left((b+\rho) \int_{t}^{t+\delta} e^{-\lambda_{k}(t-\tau)} d \tau\right)^{2}
\end{aligned}
$$

It is clear from these inequalities that we can choose $\delta$ so that $I_{3}<\varepsilon^{2} / 3$. From the estimations for $I_{1}, I_{2}, I_{3}$ obtained above we conclude according to (43) that $\|z(t+\delta)-z(t)\|_{H_{r}}<\varepsilon$, meaning that the function $z(t), 0 \leq t \leq h$, is continuous.

We now show that the problem (15)-(17) has a unique solution. We assume the contrary, that it has two different solutions $z^{\prime}(t), z^{\prime \prime}(t), 0 \leq t \leq h$ for the same functions $z_{0}(\cdot) \in$ $X$ and $u(\cdot)$. Then their difference $Z(t)=z^{\prime}(t)-z^{\prime \prime}(t), 0 \leq t \leq$ $h$, as a solution of corresponding homogeneous problem (15)(17), can be represented in the form of the Fourier series (21), and the Fourier coefficients $Z_{k}(t), 0 \leq t \leq h$, of the function $Z(t), 0 \leq t \leq h$, are solutions of the following infinite system of differential equations:

$$
\begin{array}{r}
\dot{z}_{k}(t)+\lambda_{k} z_{k}(t)=0, \quad z_{k}(0)=0, \\
0 \leq t \leq h, \quad k=1,2, \ldots
\end{array}
$$

Therefore, from the representation (29) we obtain $Z_{k}(t)=0$ on $0 \leq t \leq h$. Consequently, $Z(t)=0,0 \leq t \leq h$, a contradiction. This proves the theorem.

\section{Main results}

Consider heat exchange control problem with lag (15)-(17).

Definition 5. A set $W \subset R^{1}$ is referred to as the strong invariant set on the time interval $[0, T]$ with respect to the problem (15)-(19) if for any $z_{0}(t),\left\|z_{0}(t)\right\|_{H_{r}} \in W$, and $u(t),\|u(t)\|_{H_{r}} \leq$ $\rho, t \in[0, T]$, the inclusion $\|z(t)\|_{H_{r}} \in W$ holds true on $[0, T]$.
Definition 6. A set $W \subset R^{1}$ is referred to as the weak invariant set on the time interval $[0, T]$ with respect to the problem (15)-(19) if for any $z_{0}(t),\left\|z_{0}(t)\right\|_{H_{r}} \in W$ there exists $u(t)$, $\|u(t)\|_{H_{r}} \leq \rho, t \in[0, T]$, such that the inclusion $\|z(t)\|_{H_{r}} \in W$ holds true on $[0, T]$.

Further, strong and weak invariant sets of the form $W=$ $[0, b]$ will be investigated, where $b$ is a positive number. We will find relations among the parameters $T, b, \rho$, and $\lambda_{i}$ in order to guarantee strong or weak invariance of the set $W$ on the time interval $[0, T]$ with respect to the problem (15)-(19) (or (15)-(17), (20)).

Theorem 7. If $\lambda_{1} \geq 1$, the set $W=[0, b]$ is strong invariant on $[0, T]$ with respect to the problem (15)-(19) if and only if

$$
\rho \leq\left(\lambda_{1}-1\right) b \text {. }
$$

Proof. Let (46) hold true and let $z_{0}(t),\left\|z_{0}(t)\right\|_{H_{r}} \leq b,-h \leq t \leq$ 0 , and $u(t),\|u(t)\|_{H_{r}} \leq \rho, 0 \leq t \leq h$ be any functions. Using the same reasoning as in the proof of Theorem 4 , we get from (40) that

$$
\begin{gathered}
\|z(t)\|_{H_{r}}^{2} \leq \chi^{2}(t) ; \\
\chi(t)=e^{-\lambda_{1} t} b+a_{1}(t)(b+\rho), \quad 0 \leq t \leq h .
\end{gathered}
$$

Then

$$
\begin{gathered}
\chi(0)=b, \\
\chi^{\prime}(t)=-\lambda_{1} e^{-\lambda_{1} t} b+e^{-\lambda_{1} t}(b+\rho) \\
=e^{-\lambda_{1} t}\left(-\lambda_{1} b+b+\rho\right) \leq 0 .
\end{gathered}
$$

Hence, $\chi^{\prime}(t) \leq 0$ meaning that the function $\chi(t)$ is nonincreasing. For this reason, $\|z(t)\|_{H_{r}} \leq b, 0 \leq t \leq h$.

The same reasoning can be applied for the time interval $[h, 2 h]$ to obtain $\|z(t)\|_{H_{r}} \leq b$. For any positive number $T>0$, we can continue in this fashion to obtain

$$
\|z(t)\|_{H_{r}} \leq b, \quad 0 \leq t \leq T
$$

that is, $W$ is strong invariant.

We now turn to the proof of the second part of the theorem. Let the set $W$ be strong invariant. Suppose, contrary to our claim, that the inequality (46) fails to hold. Then, clearly, the function $\chi(t)$ is increasing. Choose the functions $z_{0}(\cdot), u(\cdot)$ as follows:

$$
\begin{gathered}
z_{01}(t-h)=\frac{b}{\lambda_{1}^{r / 2}}, \quad u_{1}(t)=\frac{\rho}{\lambda_{1}^{r / 2}}, \quad z_{0 k}(t-h)=0, \\
u_{k}(t)=0, \quad k \geq 2,0 \leq t \leq h .
\end{gathered}
$$

Then

$$
\begin{aligned}
& \|z(t)\|_{H_{r}}^{2} \\
& =\lambda_{1}^{r}\left(e^{-\lambda_{1} t} z_{01}^{0}+\int_{0}^{t} e^{-\lambda_{1}(t-\tau)}\left(z_{01}(t-\tau)+u_{1}(\tau)\right) d \tau\right)^{2} \\
& =\chi^{2}(t) .
\end{aligned}
$$


Since the function $\chi(t)$ is increasing on $[0, h]$ and $\chi(0)=b$, therefore $t, \chi(t)>b$; that is, $\|z(t)\|_{H_{r}}>b$, which contradicts the strong invariance of the set $W$. This completes the proof of Theorem 7.

Theorem 8. Let $T$ be any positive number. If $\lambda_{1} \geq 1$, the set $W=[0, b]$ is weak invariant on $[0, T]$ with respect to the problem (15)-(19).

Proof. Let $\lambda_{1} \geq 1$. Show that, under condition 9 , the set $W=$ $[0, b]$ is weak invariant with respect to the problem (15)-(17). Let $z_{0}(\cdot)$ be an arbitrary function from $X$. Set $u(\cdot)=0$. Then we obtain from the representation (29) of the solution of the problem (27) that

$$
\begin{aligned}
\|z(t)\|_{H_{r}}^{2} & \\
= & \sum_{k=1}^{\infty} \lambda_{k}^{r}\left(e^{-\lambda_{k} t} z_{0 k}^{0}+\int_{0}^{t} e^{-\lambda_{k}(t-\tau)} z_{0 k}(t-\tau) d \tau\right)^{2} \\
\leq & e^{-2 \lambda_{1} t} \sum_{k=1}^{\infty} \lambda_{k}^{r}\left|z_{0 k}^{0}\right|^{2} \\
& +2 e^{-\lambda_{1} t} \sum_{k=1}^{\infty} \lambda_{k}^{r}\left|z_{0 k}^{0}\right| \int_{0}^{t} e^{-\lambda_{k}(t-\tau)}\left|z_{0 k}(t-\tau)\right| d \tau \\
& +\sum_{k=1}^{\infty} \lambda_{k}^{r}\left(\int_{0}^{t} e^{-\lambda_{k}(t-\tau)} z_{0 k}^{0}(t-\tau) d \tau\right)^{2} \\
\leq & e^{-2 \lambda_{1} t} b^{2}+2 e^{-\lambda_{1} t} b a_{1}(t) b+a_{1}^{2}(t) b^{2} \\
= & \left(e^{-\lambda_{1} t}+a_{1}(t)\right)^{2} b^{2} .
\end{aligned}
$$

Here, we used the following inequality:

$$
\begin{aligned}
& \sum_{k=1}^{\infty} \lambda_{k}^{r}\left|z_{0 k}^{0}\right| \int_{0}^{t} e^{-\lambda_{k}(t-\tau)}\left|z_{0 k}(t-\tau)\right| d \tau \\
& \quad \leq \sqrt{\sum_{k=1}^{\infty} \lambda_{k}^{r}\left|z_{0 k}^{0}\right|^{2}} \sqrt{\sum_{k=1}^{\infty} \lambda_{k}^{r}\left(\int_{0}^{t} e^{-\lambda_{k}(t-\tau)}\left|z_{0 k}(t-\tau)\right| d \tau\right)^{2}}
\end{aligned}
$$

and Assumption 2. Since the function

$$
\xi(t)=e^{-\lambda_{1} t}+\frac{1-e^{-\lambda_{1} t}}{\lambda_{1}}, \quad 0 \leq t \leq h
$$

satisfies

$$
\xi(0)=1, \quad \xi^{\prime}(t)=\left(1-\lambda_{1}\right) e^{-\lambda_{1} t} \leq 0,
$$

we have $0<\xi(t) \leq 1$. Then in view of (52) we conclude that $\|z(t)\|_{H_{r}} \leq b, 0 \leq t \leq h$. Repeated application of this reasoning to the intervals $[j h,(j+1) h], j=1,2, \ldots$ enables us to conclude that $\|z(t)\|_{H_{r}} \leq b, 0 \leq t \leq T$, and this completes the proof.

We now turn to the problem (15)-(17), (20).
Definition 9. The set $W \subset R^{1}$ is called weak invariant on $[0, T]$ with respect to the problem (15)-(17), (20) if for any $z_{0}(t)$, $\left\|z_{0}(t)\right\|_{H_{r}} \in W, t \in[-h, 0]$ there exists $u(t),\|u(\cdot)\|_{L_{2}\left([0, T] ; H_{r}\right)} \leq$ $\rho$, such that the inclusion $\|z(t)\|_{H_{r}} \in W$ holds for all $t \in$ $[0, T]$.

Definition 10. The set $W \subset R^{1}$ is called strong invariant on $[0, T]$ with respect to the problem (15)-(17), (20) if for any $z_{0}(t),\left\|z_{0}(t)\right\|_{H_{r}} \in W, t \in[-h, 0]$ and $u(t),\|u(\cdot)\|_{L_{2}\left([0, T] ; H_{r}\right)} \leq \rho$ the inclusion $\|z(t)\|_{H_{r}} \in W$ holds for all $t \in[0, T]$.

Proposition 11. For any function $g(\tau), 0 \leq \tau \leq t \leq T$, for which $\|g(\cdot)\|_{L_{2}\left([0, T] ; H_{r}\right)} \leq N$, the following inequality holds:

$$
\begin{gathered}
\sum_{k=1}^{\infty} \lambda_{k}^{r}\left(\int_{0}^{t} e^{-\lambda_{k}(t-\tau)} g_{k}(\tau) d \tau\right)^{2} \leq a_{2}(t) N^{2}, \\
\quad 0 \leq t \leq T ; \\
a_{2}(t)=\frac{1-e^{-2 \lambda_{1} t}}{2 \lambda_{1}},
\end{gathered}
$$

where $T$ a positive number.

The proof follows from the following chain of relations

$$
\begin{aligned}
& \sum_{k=1}^{\infty} \lambda_{k}^{r}\left(\int_{0}^{t} e^{-\lambda_{k}(t-\tau)} g_{k}(\tau) d \tau\right)^{2} \\
& \quad \leq \sum_{k=1}^{\infty} \lambda_{k}^{r} \int_{0}^{t} e^{-2 \lambda_{k}(t-\tau)} d \tau \cdot \int_{0}^{t} g_{k}^{2}(\tau) d \tau \\
& \leq a_{2}(t) \sum_{k=1}^{\infty} \lambda_{k}^{r} \int_{0}^{t} g_{k}^{2}(\tau) d \tau \\
& \quad=a_{2}(t)\|g(\cdot)\|_{L_{2}\left([0, t] ; H_{r}\right)}^{2} \leq a_{2}(t) N^{2} ;
\end{aligned}
$$

here we used the Cauchy-Schwartz inequality and the relation $e^{-\lambda_{k} t} \leq e^{-\lambda_{1} t}, t \geq 0, k=1,2, \ldots$..

Theorem 12. If $\rho>0$, then the set $W=[0, b]$ is not strong invariant with respect to the problem (15)-(17), (20) on $[0, T]$, where $T$ is any number.

Proof. Let $\rho>0$. To prove the theorem, we use the fact that modulus of the admissible control function can take any big values on small time interval. Consider the function

$$
\eta(t)=e^{-\lambda_{1} t} b+\frac{1-e^{-\lambda_{1} t}}{\lambda_{1}} b+e^{-\lambda_{1} t} \sqrt{t} \rho, \quad t \geq 0 .
$$

Note that $\eta(0)=b$ and $\eta(t)>0$ for all $t>0$. Assuming $t>0$, we have

$$
\eta^{\prime}(t)=e^{-\lambda_{1} t}\left(b-\lambda_{1} b-\lambda_{1} \rho \sqrt{t}+\frac{\rho}{2 \sqrt{t}}\right) .
$$

Due to the term $e^{-\lambda_{1} t}(\rho / 2 \sqrt{t})$, we obtain that there exists $t_{1}$, $0<t_{1}<h$, such that $\eta^{\prime}(t)>0$ whenever $0<t \leq t_{1}$. 
We now specify the initial function and admissible control function on $[0, h]$ as follows:

$$
\begin{aligned}
& z_{0}(\tau-h)=\frac{b}{\lambda_{1}^{r / 2}} \varphi_{1}, \quad 0 \leq \tau \leq h, \\
& u(\tau)= \begin{cases}\frac{\rho}{\lambda_{1}^{r / 2} \sqrt{t_{1}}} \varphi_{1}, & 0 \leq \tau<t_{1}, \\
0, & t_{1} \leq \tau \leq h .\end{cases}
\end{aligned}
$$

We observe that

$$
\begin{gathered}
\left\|z_{0}(\tau)\right\|_{H_{r}} \leq b, \quad-h \leq \tau \leq 0, \\
\|u(\cdot)\|_{L_{2}\left([0, h] ; H_{r}\right)} \leq \rho .
\end{gathered}
$$

Then by the representation (30) we have

$$
\begin{array}{r}
z(t)=\left(e^{-\lambda_{1} t} \frac{b}{\lambda_{1}^{r / 2}}+\int_{0}^{t} e^{-\lambda_{1}(t-\tau)}\left(\frac{b}{\lambda_{1}^{r / 2}}+\frac{\rho}{\lambda_{1}^{r / 2} \sqrt{t_{1}}}\right) d \tau\right) \varphi_{1}, \\
0 \leq t \leq t_{1} .
\end{array}
$$

Hence

$$
\begin{aligned}
& \|z(t)\|_{H_{r}}^{2} \\
& =\lambda_{1}^{r}\left(e^{-\lambda_{1} t} \frac{b}{\lambda_{1}^{r / 2}}+\int_{0}^{t} e^{-\lambda_{1}(t-\tau)}\left(\frac{b}{\lambda_{1}^{r / 2}}+\frac{\rho}{\lambda_{1}^{r / 2} \sqrt{t_{1}}}\right) d \tau\right)^{2}, \\
& 0 \leq t \leq t_{1} .
\end{aligned}
$$

This implies that

$$
\begin{aligned}
\left\|z\left(t_{1}\right)\right\|_{H_{r}} & \\
= & e^{-\lambda_{1} t_{1}} b+b \int_{0}^{t_{1}} e^{-\lambda_{1}\left(t_{1}-\tau\right)} d \tau \\
& +\int_{0}^{t_{1}} e^{-\lambda_{1}\left(t_{1}-\tau\right)} \frac{\rho}{\sqrt{t_{1}}} d \tau \\
\geq & e^{-\lambda_{1} t_{1}} b+b \int_{0}^{t_{1}} e^{-\lambda_{1}\left(t_{1}-\tau\right)} d \tau \\
& +\int_{0}^{t_{1}} e^{-\lambda_{1} t_{1}} \frac{\rho}{\sqrt{t_{1}}} d \tau=\eta\left(t_{1}\right) .
\end{aligned}
$$

Since $\eta(0)=b$ and $\eta^{\prime}(t)>0$ for $0<t \leq t_{1}$, then $\eta\left(t_{1}\right)>b$. Therefore $\left\|z\left(t_{1}\right)\right\|_{H_{r}}>b$ which shows that the set $W=[0, b]$ is not strong invariant, and the proof of Theorem 12 is complete.

Theorem 13. If $\lambda_{1} \geq 1$, then the set $W=[0, b]$ is weak invariant with respect to the problem (15)-(17), (20) on $[0, h]$.

The proof of the theorem is similar to that of Theorem 8 .

\section{Conflict of Interests}

The authors declare that there is no conflict of interests regarding the publication of this paper.

\section{Acknowledgment}

The present research was partially supported by the National Fundamental Research Grant Scheme FRGS of Malaysia (no. 01-01-13-1228FR).

\section{References}

[1] Kh. G. Guseı̆nov and V. N. Ushakov, "Strongly and weakly invariant sets with respect to a differential inclusion," Doklady Akademii Nauk USSR, vol. 303, article 4, pp. 794-796, 1988.

[2] J.-P. Aubin, "A survey of viability theory," SIAM Journal on Control and Optimization, vol. 28, no. 4, pp. 749-788, 1990.

[3] A. B. Kurzhanski and T. F. Filippova, "On the description of the set of viable trajctories of a control system," Differential Equations, vol. 23, no. 8, pp. 1303-1315, 1987.

[4] A. Z. Fazilov and U. M. Ibragimov, On the Strong Invariant Sets of Linear Control System, vol. 4 of Mathematics, Informatics, and Physics, Science and Education of South Kazakhstan, 2003.

[5] G. Haddad, "Monotone trajectories of differential inclusions and functional-differential inclusions with memory," Israel Journal of Mathematics, vol. 39, no. 1-2, pp. 83-100, 1981.

[6] A. H. Borzabadi, A. V. Kamyad, and M. H. Farahi, "Optimal control of the heat equation in an inhomogeneous body," Journal of Applied Mathematics and Computing, vol. 15, no. 1-2, pp. 127-146, 2004.

[7] H. O. Fattorini, "The maximum principle for control systems described by linear parabolic equations," Journal of Mathematical Analysis and Applications, vol. 259, no. 2, pp. 630-651, 2001.

[8] G. G. Islamov and Yu. V. Kogan, "The differential-difference problem of control of diffuzion process," Vestnik Udmurtskogo Universiteta, vol. 303, no. 1, pp. 121-126, 2008.

[9] M. Tukhtasinov and U. Ibragimov, "Sets invariant under an integral constraint on controls," Russian Mathematics, vol. 55, no. 8, pp. 59-65, 2011.

[10] M. Tukhtasinov and U. Ibragimov, "Invariant sets with respect to the system with lag," Reports of Academy of Sciences of Republic of Uzbekistan, vol. 15, article no. 6, pp. 12-15, 2011.

[11] Sh. Alimov, "On the null-controllability of the heat exchange process," Eurasian Mathematical Journal, vol. 2, article no. 3, pp. 5-19, 2011.

[12] S. Albeverio and Sh. Alimov, "On a time-optimal control problem associated with the heat exchange process," Applied Mathematics \& Optimization, vol. 57, no. 1, pp. 58-68, 2008.

[13] S. G. Mikhlin, Linear Partial Differential Equations, Vysshaya Shkola, Moscow, Russia, 1977.

[14] S. A. Avdonin and S. A. Ivanov, Controllability of Systems with Distributed Parameters and Families of Exponents, UMKVO, Kiev, Ukraine, 1989. 


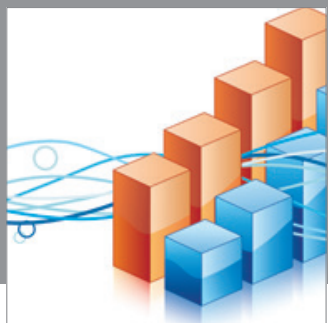

Advances in

Operations Research

mansans

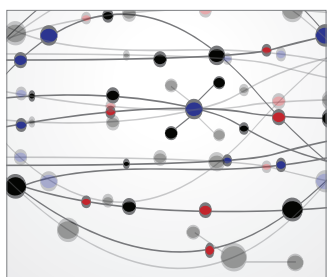

The Scientific World Journal
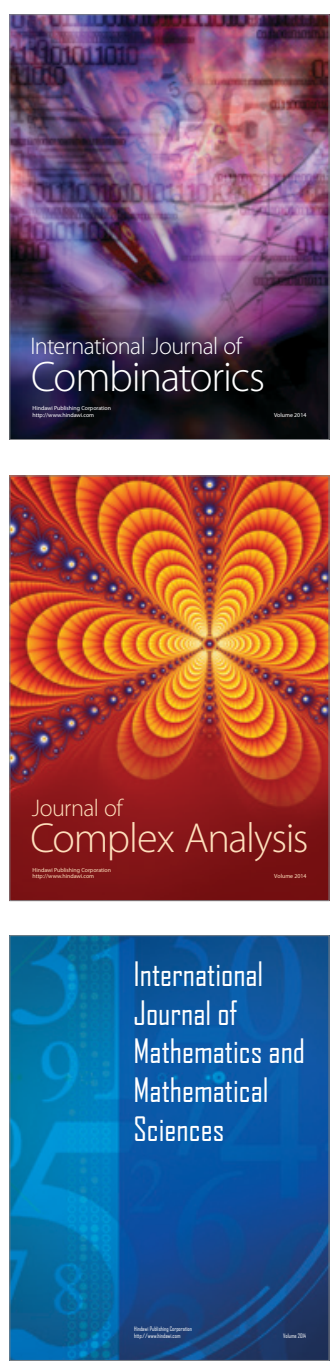
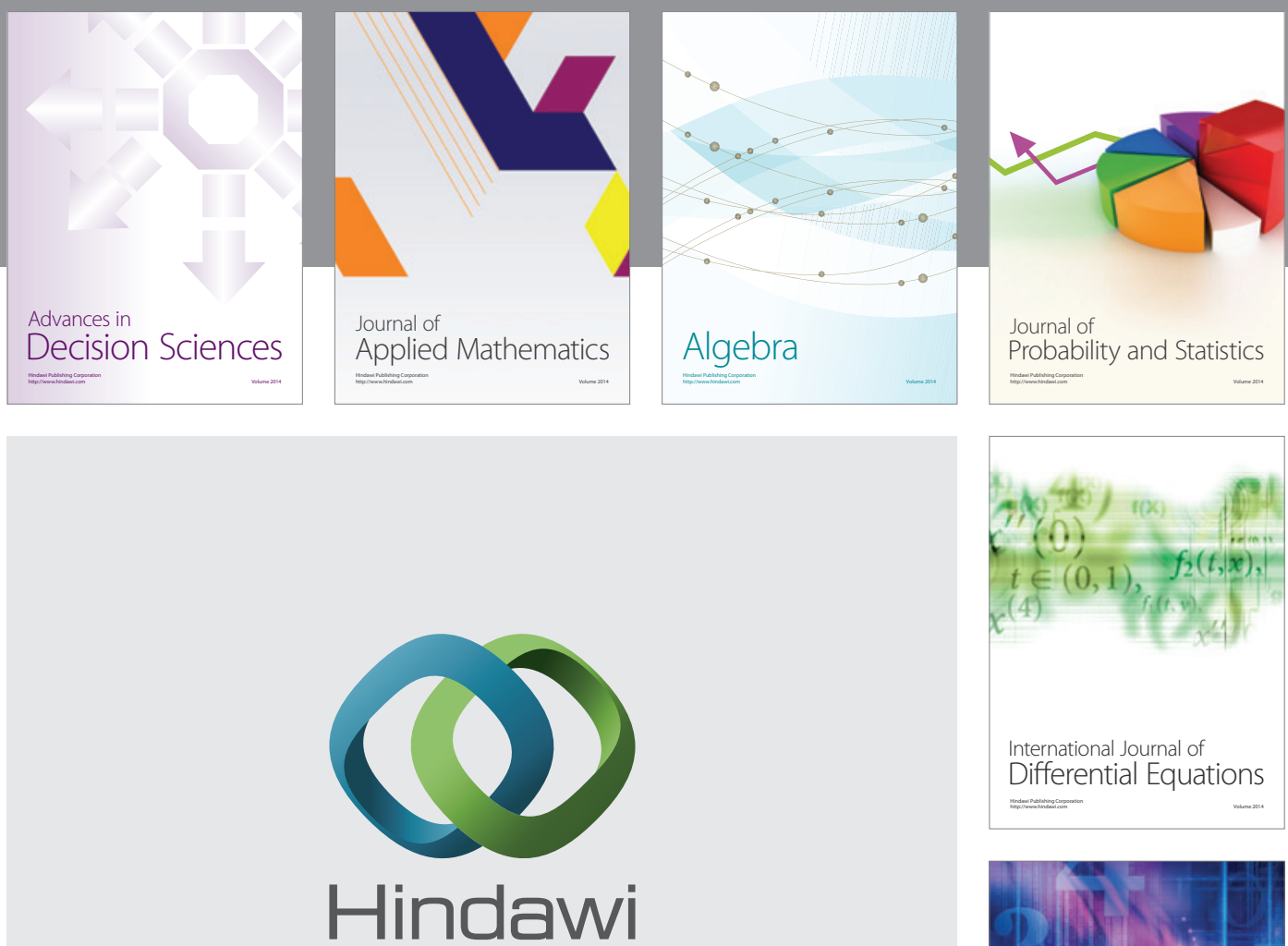

Submit your manuscripts at http://www.hindawi.com
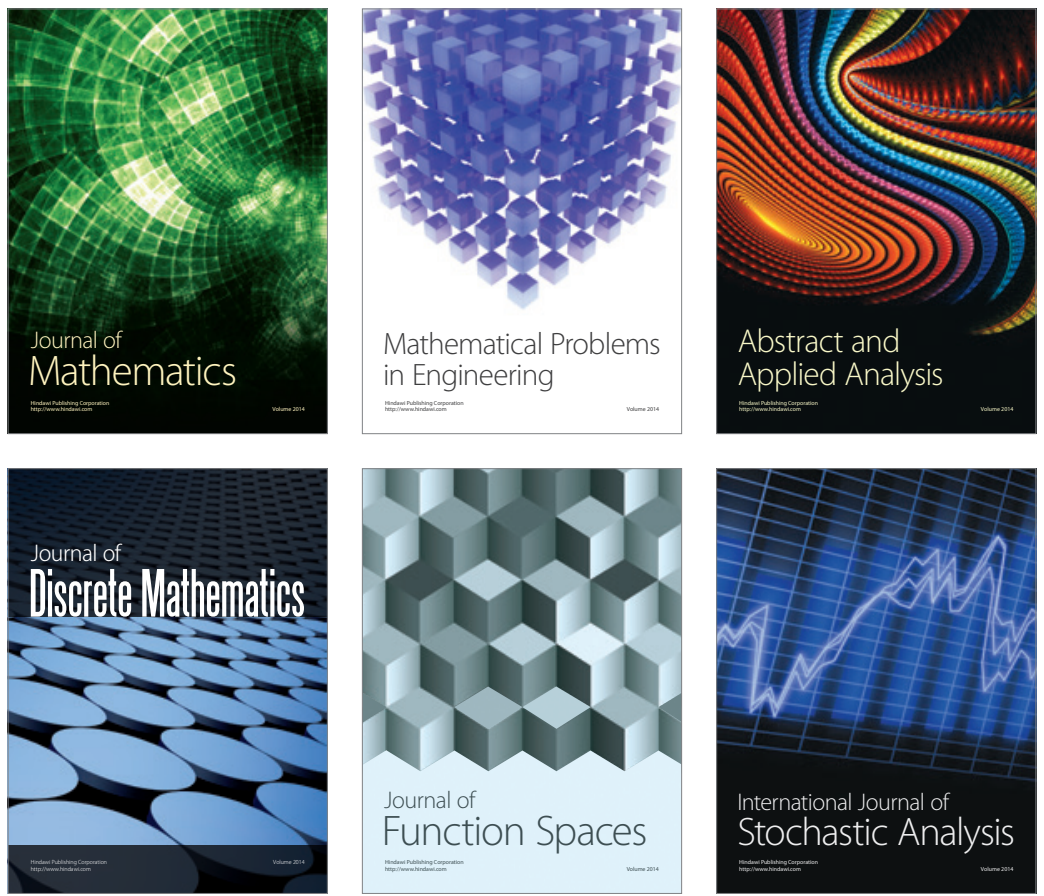

Journal of

Function Spaces

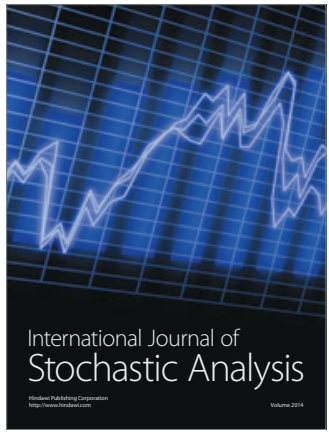

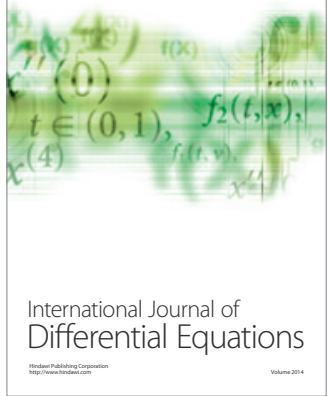
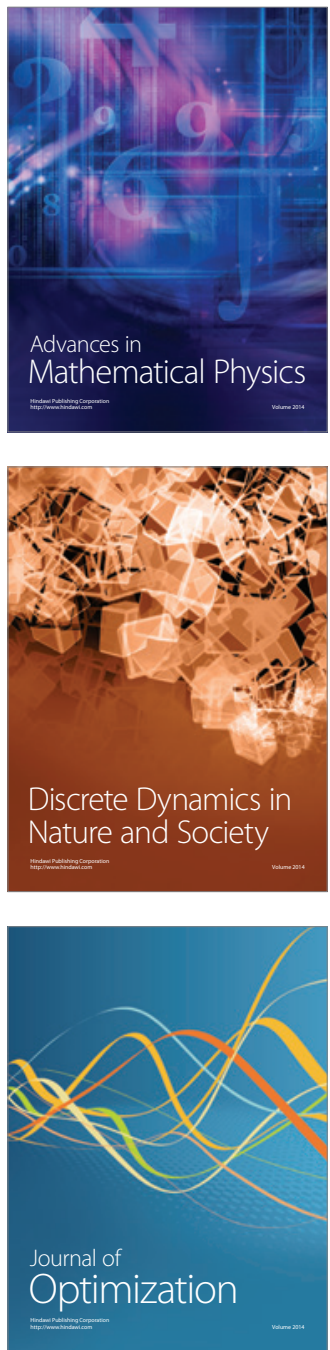